\title{
Preparation and Modification of Chitosan-based Membrane
}

\author{
Miao Sun, ${ }^{\mathrm{a}, 1}$ Lincheng Yuan, ${ }^{\mathrm{a}, 1}$ Xiaobin Yang ${ }^{\mathrm{a}, \mathrm{b}, 1}$ and Lu Shao ${ }^{\mathrm{a} *}$
}

\begin{abstract}
Chitosan (CS) has been studied worldwide, including its recent applications in membrane science. Despite its advantages, drawbacks such as weak mechanical property and low solubility hampered its wide range of applications. Herein, CS films with various deactivation degree (DD) and CS/poly(ethylene oxide) (PEO) blend film (PEO with different molecular weights) are prepared, and vapor-crosslink technique is applied to crosslink various CS based membranes using glutaraldehyde. With the introduction of PEO into CS, the blend membrane surface is transformed from a dense structure to a porous structure. The formation of networks in cross-linked CS/PEO membranes by glutaraldehyde decreased the pore size as well as the thermal stability of cross-linked CS/PEO. Besides, a gradual increase in the tensile strength with the increasing cross-linking time is observed initially. Our work exhibits the great potential of CS-based membrane modified by PEO and glutaraldehyde.
\end{abstract}

Keywords: Chitosan; Blending; Cross-linking; Chemical and physical property.

Received: 21 April 2020; Accepted: 08 June 2020.

Article type: Research article.

\section{Introduction}

Chitosan [CS, (1-4)-2-amino-2-deoxy-D-glucon] is a natural polyaminosaccharide obtained from chitin and consists of glucosamine and $\mathrm{N}$-acetylglucosamine units. Its low cost, nontoxicity, degradability, and biocompatibility have qualified its potential in medicine, food and biology researches..$^{[1-4]}$ Neat CS products with some shortcomings, viz. only soluble in acid environment and weak mechanical property are seriously limited in various applications.

Researchers have devoted to the modification of CS to extend its application scopes. For example, the solubility of CS in water has received massive interest to improve the processing properties..$^{[5-8]}$ Generally, the amino and hydroxyl groups in CS are utilized for modification with physical or chemical methods. By blending with 3-amino siloxane oligomer ${ }^{[9]}$ polylactide, ${ }^{[10]}$ polyethylene glycol $(\mathrm{PEG}){ }^{[11]}$ or albumin, ${ }^{[12]}$ enhanced physicochemical properties of CS have been achieved. For example, Kolhe et al..$^{[1]}$ blended and copolymerized PEG with CS to improve the ductility of CS films. An improvement in ductility was obtained for all compositions of the blend as manifested by a decrease in

\footnotetext{
a State Key Laboratory of Urban Water Resource and Environment, School of Chemistry and Chemical Engineering, Harbin Institute of Technology, Harbin 150001, China

${ }^{b}$ Chemical Sciences and Engineering Division, Argonne National Laboratory, Lemont, IL 60439, United States

*E-mail:shaolu@hit.edu.cn (L.Shao).

${ }^{1}$ These authors contributed equally to this work.
}

modulus and an increase in strain at break. More chemical modification techniques ${ }^{[13-24]}$ have achieved fairly good results. Besides, the preparation of chitosan nano-composites ${ }^{[25-28]}$ with various nanomaterials is a new way that attracts increasing interests.

Poly(ethylene oxide) (PEO) is a water-soluble polymer and exhibits many useful properties, such as low toxicity, hydrophilicity, high viscosity, ability to form hydrogen bonds, and biocompatibility with other bioactive substance and immunogenicity. Besides, it is available at different molecular weights. A combination of these two polymers may have beneficial effects on the biological characteristics of the resulting membrane when considering the useful biological activities of CS and PEO. ${ }^{[29]}$ In this study, CS films are modified by blending with PEO, and then glutaraldehyde is utilized to carry out the cross-linking reaction. This work mainly studies the preparation process of CS functional film, the modification methods of CS functional film, and explores the effect of both degree of deacetylation (DD) and crosslinking time. Expectedly, through this work, the optimized preparation system and conditions of the CS functional membrane are determined, and the physical structure and chemical properties of the membrane material are improved. Fourier Transform infrared (FTIR) measurements, atomic force microscope (AFM), differential scanning calorimetry (DSC), and thermogravimetric analysis (TGA), etc. are performed to characterize the alterations of CS properties and analyze the micro-mechanism of such influences. We 
expected some insights into the development of CS films with better structures and extensive applications of CS based membranes.

\section{Experimental}

\subsection{Materials}

CS with different DD $(86.11 \%$, and $93.48 \%)$ was supplied by Jinke Biological Ltd (Zhejiang, China). Polyethylene oxide (PEO) with various average molecular weights (i.e., 400, $2000+10 \%, 20000+10 \%$ ) was purchased from Yili Chemicals Ltd. Glutaraldehyde was obtained from Boli Chemical Ltd (Tianjin, China). All other common reagents were supplied by local companies. All the reagents were used as received.

\subsection{Determination of chitosan's solvable property}

CS films used in this study were prepared using the solvent casting method. In order to find the suitable concentration for the fabrication of CS films, CS (with DD of $86.11 \%$, and $93.48 \%$ ) and acetic acid (2 wt\%) were used to prepare CS solution of various concentrations: $0.5 \%, 1 \%, 2 \%, 5 \%$ and $10 \%$. Based on the primary experimental exploration, we found the following facts.

(1) CS with a higher DD exhibited a better solvable property in an acid solvent, but the improvement in degree was not so significant.

(2) The viscosity of CS solution increased with increasing the concentration. Thus, the CS solutions with concentrations of $5 \%$ and $10 \%$ resulted in bad fluidity, while the ones of $0.5 \%$, $1 \%$ and $2 \%$ could flow pretty well.

(3) The stirring procedure led to the appearance of air bubbles, and it became more obvious when increasing the CS concentrations. So, efforts should be made to diminish them in case they influenced the property of films.

Concerning all the above factors, $0.5 \%$ CS solution dissolved by $1 \%$ acetic acid was utilized in this study.

\subsection{Preparation of CS film and CS/PEO film}

CS $(0.33 \mathrm{~g})$ was dissolved in $63 \mathrm{~mL}$ of $1 \mathrm{wt} \%$ acetic acid solution. The CS solution was stirred overnight at room temperature. After filtration, the PEO solution $(0.11 \mathrm{~g}$ PEO was dissolved in $2 \mathrm{~mL}$ distilled water) was added into the aforementioned CS system. This solution was then stirred overnight at room temperature. This mixture was sonicated for 30 min using an ultrasonic generator (Model KQ-100DB) and stood overnight for de-gassing. Finally, the CS/PEO blending film was obtained via solution casting and dried under vacuum at $20^{\circ} \mathrm{C}$ overnight.

Preparation of blank CS film had a similar process without adding PEO. Any further tests were carried out after the physically absorbed solvent-HAc was removed.

\subsection{Preparation of crosslinked CS/PEO film}

First, the CS/PEO films were fixed onto a holder, which was then put into a beaker. Second, a certain amount of glutaraldehyde was added into the beaker so that the CS/PEO films could reach above the liquid level. Third, aluminum foil was utilized to cover the beaker. Finally, the beaker was transferred to a water bath maintained at $25{ }^{\circ} \mathrm{C}$, fumigating $\mathrm{CS} / \mathrm{PEO}$ films with glutaraldehyde vapor for $0.5,1,1.5$ and 2 $\mathrm{h}$, respectively.

We found that as the cross-linking reaction went on, the transparent CS/PEO films gradually changed into pale yellow, then to deep yellow. Meanwhile, the films became stiff and harder.

\subsection{Characterization}

The infrared spectrum of PEO solids was measured by the potassium bromide tablet method of Nicolet-Nexus 670 Fourier Transform Infrared spectrometer, and the infrared spectrum of various films was measured by total reflection method. The measurement range was $400-4000 \mathrm{c} \mathrm{m}^{-1}$; the number of scans was 32; the resolution was $4 \mathrm{~cm}^{-1}$. Thermal properties were tested with a NETZSCH STA449C at a heating rate of $10{ }^{\circ} \mathrm{C} / \mathrm{min}$ under nitrogen atmosphere. The surface morphology of films was obtained with an atomic force microscope from Russia at room temperature.

\subsection{Membrane Performance}

Tensile property tests were conducted with a WD-1 universal testing machine (Changchun Second Material Testing Machine Factory, China) at an elongation rate of 20 $\mathrm{mm} / \mathrm{min}$. The tensile strength of films was calculated by Equation (1):

$$
\delta=F / D \times S
$$

where $\delta$ is the tensile strength of film (MPa); $F$ is the force when broken $(\mathrm{N}) ; D$ is the thickness of the film $(\mu \mathrm{m})$ and $S$ is the width of films (mm).

Water absorbing examination was carried out as follows. At first, CS film samples were dried under vacuum at $20{ }^{\circ} \mathrm{C}$ for $24 \mathrm{~h}$, and the mass as the original mass $\left(\mathrm{W}_{\mathrm{s}}\right)$ was noted down. Then the remaining $\mathrm{HAc}$ with $\mathrm{NaOH}$ diluted solution was removed. The samples were immersed into distilled water for $48 \mathrm{~h}$ to achieve swelling equilibrium, and the mass now as the final mass $\left(\mathrm{W}_{\mathrm{d}}\right)$ was noted down. The relative water intake capacity $(H)$ was calculated by Equation (2):

$$
\mathrm{H}=\left(W_{s}-W_{d}\right) / W_{s} \times 100 \%
$$

The cross-linking density ( $\rho)$ of neat CS film was shown as Equation (3):

$$
\rho=1 / M_{c}
$$

where $M c$ is the average molecular weight between the neighboring cross-linking points. It was carried out by dilatometer method and calculated by the Flory-Rehner Equation as shown by Equation (4):[30]

$$
-\left[\ln \left(1-u_{r}\right)+\chi \times u_{r}^{2}\right]=\rho V_{0} M_{c}^{-1} u_{r}^{1 / 3}
$$

where $\chi$ is the interacting constant between $\mathrm{CS}$ and $\mathrm{H}_{2} \mathrm{O}, \rho$ is the density of CS, $V_{m}$ is the molar volume of $\mathrm{H}_{2} \mathrm{O}$, and $u_{r}$ stands for the volume fraction of the expended CS. $u_{r}$ is calculated by Equation (5):

$$
\mu_{r}=\left(W_{d} \times 1.003\right) /\left[W_{d} \times 1.003\right]+\left(W_{s}-W_{d}\right) \times 1.25(5)
$$




\section{Results and Discussion}

3.1 Possible underlying mechanism during the crosslinking process

The cross-linking mechanism between CS and glutaraldehyde can be interpreted that the amino groups in CS react with one or two glutaraldehyde's terminal aldehyde groups (Fig. 1), the interconnected network structure is formed inside CS, significantly affecting its physicochemical properties.
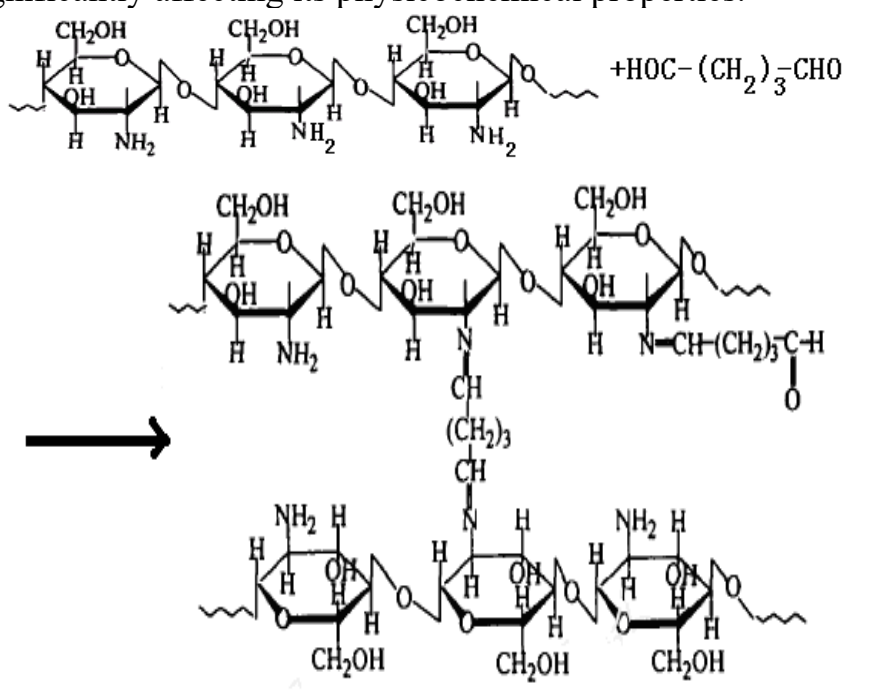

Fig.1 Possible cross-linking mechanism of CS and glutaraldehyde.

\subsection{Surface chemistry characterization of membranes}

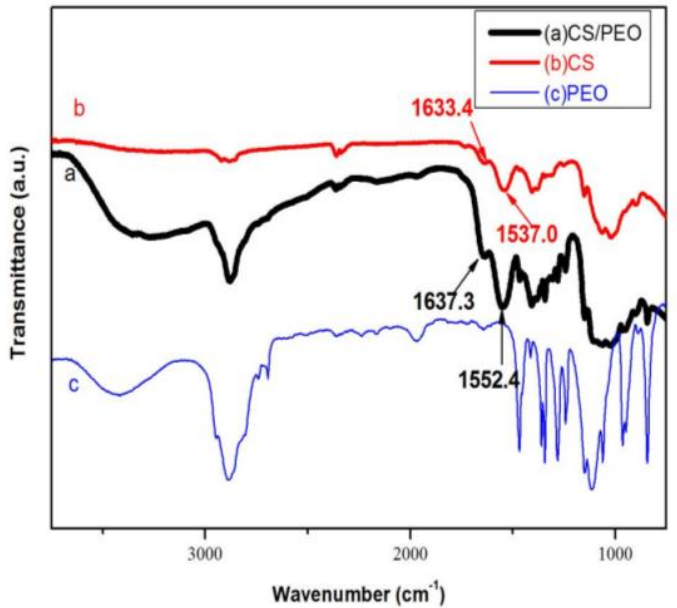

Fig. 2 FTIR spectra of (a) PEO2000, (b) CS (with DD of 86.11\%), (c) CS/PEO film.

To characterize the chemical structures of pure CS film and CS/PEO blend films, the FTIR spectra of PEO (PEO2000), CS (with DD of $86.11 \%$ ), and CS/PEO film are obtained and depicted in Fig. 2. According to Fig. 2, the spectrum of $\mathrm{CS} / \mathrm{PEO}$ film, without the appearance of new peaks and disappearance of original peaks, was just a physical superposition of PEO and CS spectrum, indicating that the blend of PEO and CS is only a physical process, and no chemical reaction is involved. Besides, $\mathrm{CS}$ has similar chemical and spatial structures to PEO (CS has plenty of lateral hydroxyl and $\mathrm{C}=\mathrm{O}$ in 3 and 6-carbon, while $\mathrm{PEO}$ has a a few of C-O groups and terminal hydroxyl groups), so the strong interaction of PEO and CS should be attributed to the intermolecular hydrogen bonding. The typical absorption peaks of $\mathrm{CS}$ are observed at $1633.4 \mathrm{~cm}^{-1}(\mathrm{C}=\mathrm{O}$ groups $), 1537.0$ $\mathrm{cm}^{-1}$ (N-H groups), $1378.9 \mathrm{~cm}^{-1}$ (deformation vibration peak of $\mathrm{CH}_{3}$ groups), $1344.2 \mathrm{~cm}^{-1}$ and $2883.1 \mathrm{~cm}^{-1}$ (bending and stretching vibration of $\mathrm{C}-\mathrm{N}$, respectively) in the pristine $\mathrm{CS}$ spectra. For the spectra of $\mathrm{CS} / \mathrm{PEO}$ films, the peaks of $\mathrm{C}=\mathrm{O}$ and N-H moved to high wavenumbers of 1637.3 and 1552.4 $\mathrm{cm}^{-1}$, respectively, as well as shifts of the stretching vibration peaks of $\mathrm{N}-\mathrm{H}$ and $\mathrm{O}-\mathrm{H}$ (moved to $3280.4 \mathrm{~cm}^{-1}$ from 3255.3 $\mathrm{cm}^{-1}$ and become stronger). This illustrates the enhancement of hydrogen bonding. ${ }^{[31]}$ The analysis reveals that the strong interaction does exist between PEO and CS molecular in the composite and good compatibility has been achieved.

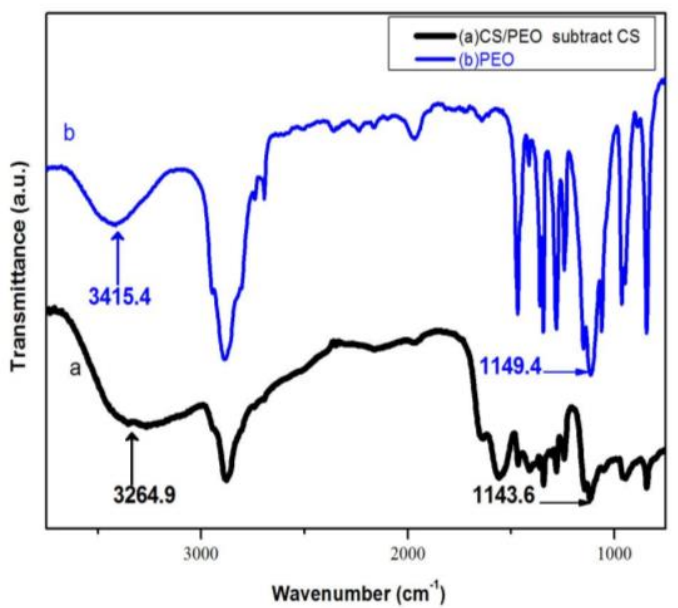

Fig. 3 FTIR spectra of (a) CS/PEO subtracting CS, and (b) PEO.

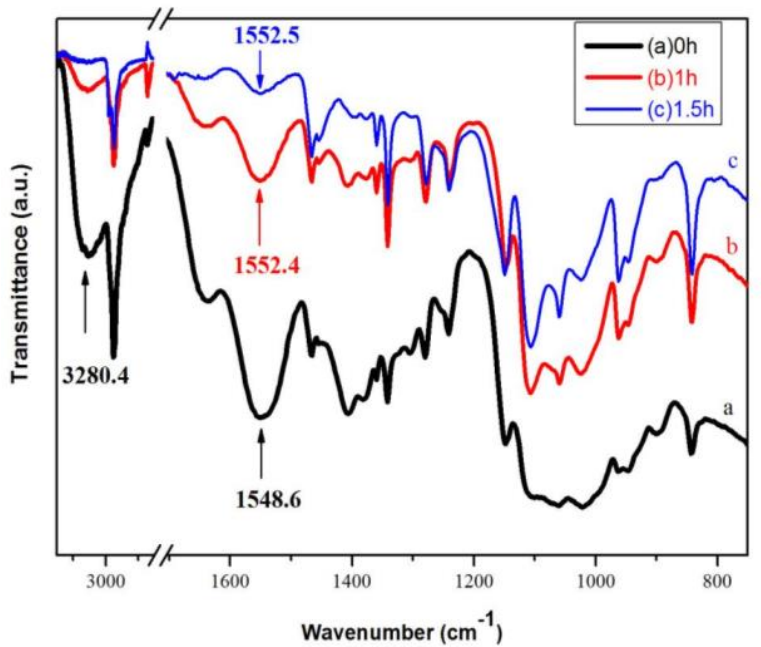

Fig. 4 FTIR spectra of the crosslinked CS/PEO 2000 film by $86.11 \%$ DD after different reaction times.

To obtain a further investigation of the interaction between CS and PEO, the subtractive spectrum and the corresponding results are shown in Fig. 3 and Table 1, respectively. Compared to blank PEO spectra, no obvious changes were 
found in the PEO subtractive spectra, demonstrating no involved chemical reaction. The decrease of $\mathrm{O}-\mathrm{H}$ and $\mathrm{C}-\mathrm{O}$ peak wavenumber is shown in Table. 1 (move to low wave number by $150.5 \mathrm{~cm}^{-1}$ and $5.8 \mathrm{~cm}^{-1}$, respectively) should also be attributed to the intermolecular hydrogen bonding between $\mathrm{CS}$ and PEO.

Table 1. The decrease of peak value of O-H and C-O bond.

\begin{tabular}{c|c|c|c|c}
\hline \multirow{2}{*}{ Sample } & \multicolumn{2}{|c|}{ O-H bond } & \multicolumn{2}{c}{ C-O bond } \\
\cline { 2 - 4 } & $\Delta\left(\mathrm{cm}^{-1}\right)$ & $\Delta \delta\left(\mathrm{cm}^{-1}\right)$ & $\Delta\left(\mathrm{cm}^{-1}\right)$ & $\Delta \delta\left(\mathrm{cm}^{-1}\right)$ \\
\hline PEO & 3415.4 & & 1149.4 & \multirow{2}{*}{-5.8} \\
\hline $\begin{array}{c}\text { CS/PEO } \\
\text { subtract CS }\end{array}$ & 3264.9 & -150.5 & 1143.6 & \\
\hline
\end{tabular}

The spectra of cross-linked CS/PEO film (the DD of CS is $86.11 \%$, and PEO2000) are tested and displayed in Fig. 4. There is no change in curve shape after cross-linking, except the N-H vibration peak (at $1548.6 \mathrm{~cm}^{-1}$ before cross-linking) which is increased by about $4 \mathrm{~cm}^{-1}$ and becomes greatly weaker at the same time. Meanwhile, the N-H vibration peak gets weaker as the cross-linking time increases. This can be explained by the decrease of amino numbers in CS after crosslinking, which results from the chemical reactions between amino and glutaraldehyde. Besides, the $\mathrm{N}-\mathrm{H}$ and $\mathrm{O}-\mathrm{H}$ stretching vibration peak (at $3280.4 \mathrm{~cm}^{-1}$ before cross-linking) show similar changes to $\mathrm{N}-\mathrm{H}$ at $1548.6 \mathrm{~cm}^{-1}$ for the same reason.

To qualify the analysis of the aforementioned cross-linking situation of CS/PEO film above. PEO20000 and CS with DD of $93.48 \%$ are applied to prepare cross-linked CS/PEO films, and FTIR tests are performed (Fig. 5 and Fig. 6). The same changes are achieved.

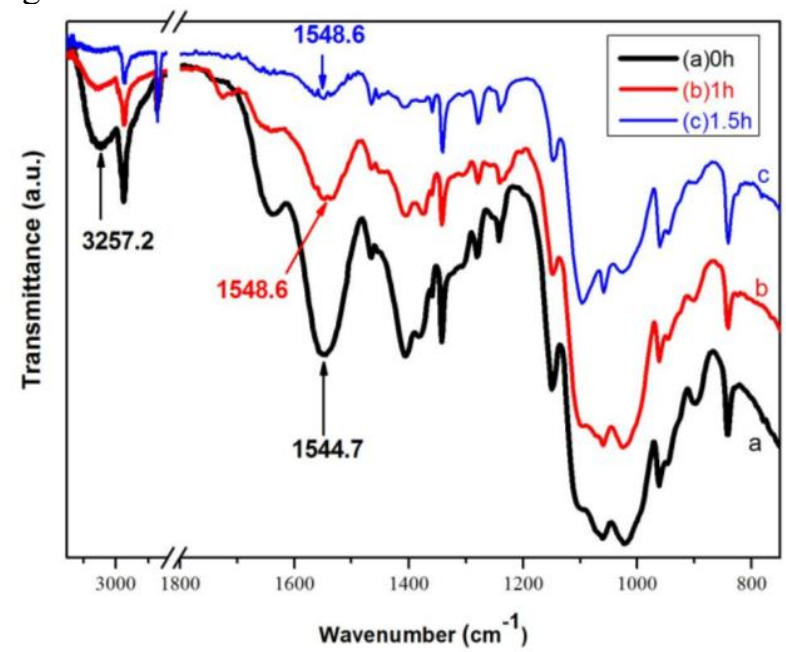

Fig. 5 FTIR spectra of the crosslinked CS/PEO 20000 film by $86.11 \%$ DD after different cross-linking time.

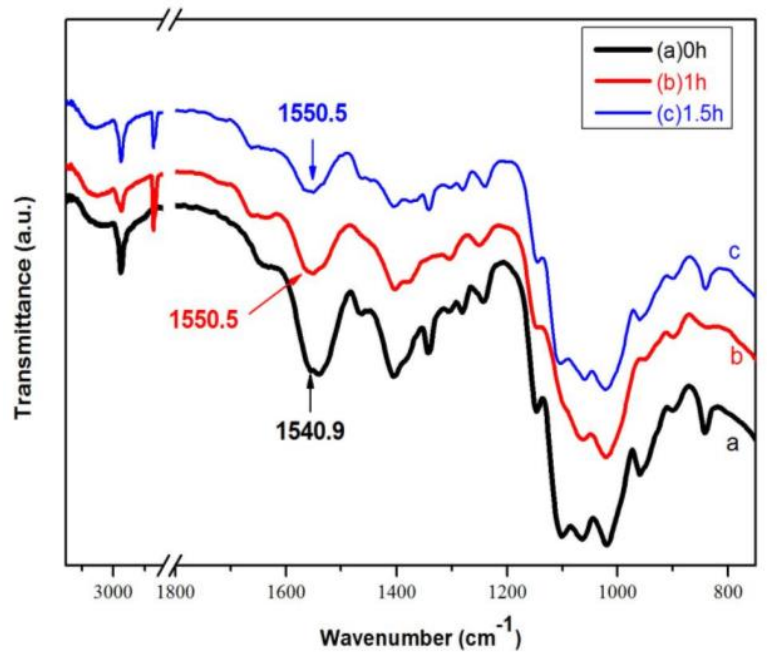

Fig. 6 FTIR spectra of the crosslinked CS/PEO 20000 film by 93.48\% DD after different cross-linking time.

\subsection{Thermal stability}

Fig. 7 shows the differential scanning calorimetric curves of $\mathrm{CS} / \mathrm{PEO}$ film and crosslinked CS/PEO films (the DD of CS is 93.48\%, and PEO2000). For CS/PEO film, the peaks appear at $54.8{ }^{\circ} \mathrm{C}$ (melting endothermic peak), $99.8{ }^{\circ} \mathrm{C}$ (evaporation endothermic peak), $310.0^{\circ} \mathrm{C}$ (decomposition exothermic peak) and $412{ }^{\circ} \mathrm{C}$ (decomposition exothermic peak of PEO). For the crosslinked CS/PEO film, it is different. First, the temperature of the $\mathrm{H}_{2} \mathrm{O}$ evaporation peak is decreased to $81.2{ }^{\circ} \mathrm{C}$. The reason may be the consumption of amino groups during the cross-linking which destroyed the intermolecular hydrogen bonding between $\mathrm{CS}$ and $\mathrm{H}_{2} \mathrm{O}$ molecules, thus the interaction was weakened, and $\mathrm{H}_{2} \mathrm{O}$ molecules could get out of the $\mathrm{CS} / \mathrm{PEO}$ blend system more easily. Another explanation is that the network structure in the crosslinked film facilitates the $\mathrm{H}_{2} \mathrm{O}$ evaporation. Second, the melting peak of PEO disappeared, while the decomposition peak at $417.0{ }^{\circ} \mathrm{C}$ remained. Maybe the latter peak had coincided with the evaporation peak of $\mathrm{H}_{2} \mathrm{O}$. Third, the decomposition exothermic peak of $\mathrm{CS}$ is decreased to $307.0^{\circ} \mathrm{C}$, indicating the decrease of the thermal stability, which is consistent with the results of Gliko-Kabir, Penhasi and Rubinstein. ${ }^{[32]}$ They found that the thermal stability of crosslinked GG depended on the amount of GA used. Excess amount of the crosslinker GA will reduce the stability of crosslinked GG. In our study, this could be explained by a larger molecular structure formed inside the CS system after cross-linking. This new structure influenced the original hydrogen bonding, further partly broke the regularity of the system, and led to the decline of thermal stability. 

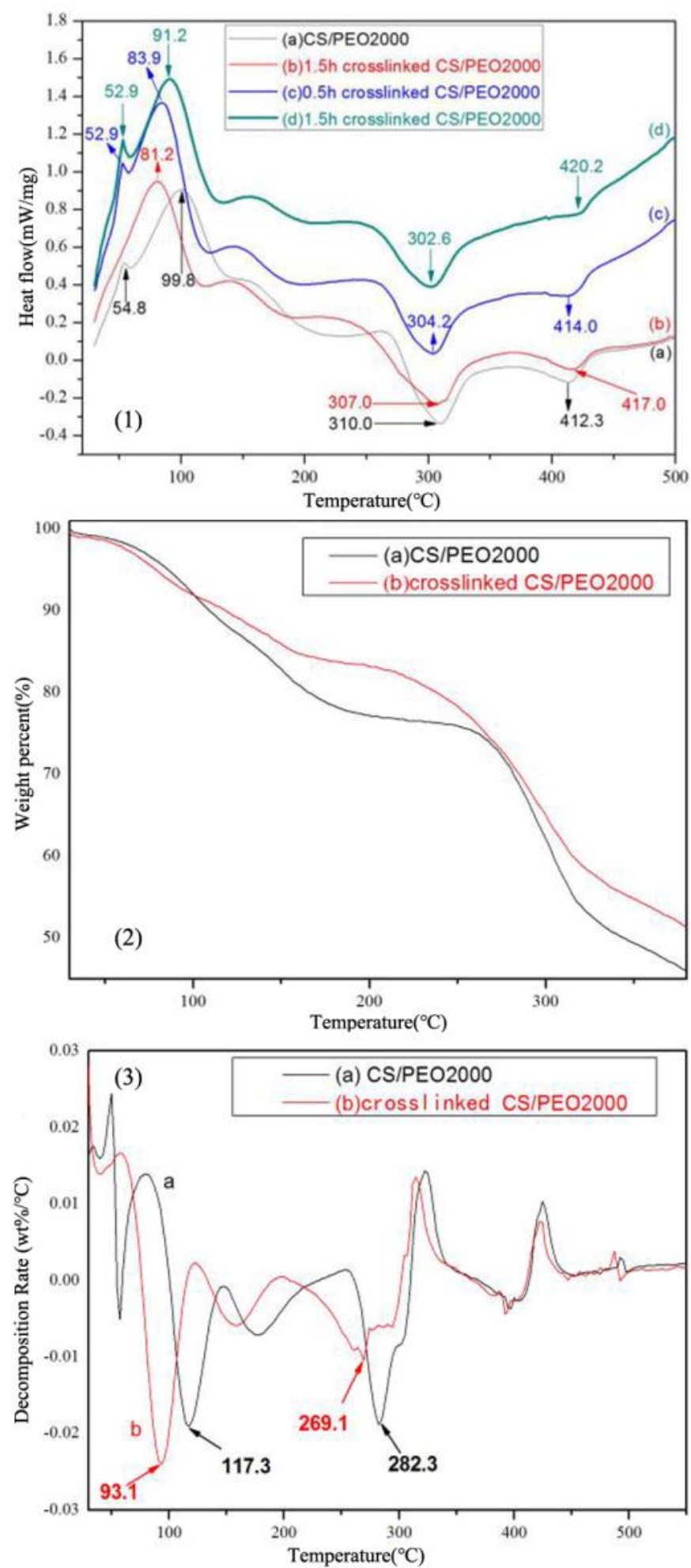

Fig. 7 Thermal property of different samples. (1) DSC curves of $\mathrm{CS} / \mathrm{PEO}$ film and crosslinked CS/PEO film, (a-b) the DD of CS is $93.48 \%$, and PEO2000, (c-d) the DD of CSis $86.11 \%$, and PEO2000. (2)-(3) TGA and DTG curves of CS/PEO and CS/PEO film and crosslinked CS/PEO film (the DD of CS is $93.48 \%$, and PEO2000)

\subsection{Surface morphology of films}

To learn more about the influence of cross-linking on the thermal property of $\mathrm{CS} / \mathrm{PEO}$ film, $0.5 \mathrm{~h}$ and $1.5 \mathrm{~h}$ crosslinked films (CS with DD of $86.11 \%$, and PEO2000) are prepared and tested with DSC (Fig. 7). The two curves exhibit a PEO melting peak at $52.9{ }^{\circ} \mathrm{C}$ in common. However, for the evaporation peak of $\mathrm{H}_{2} \mathrm{O}, 1.5 \mathrm{~h}$ crosslinked film has a higher temperature than that of $0.5 \mathrm{~h}$ crosslinked film $\left(7.3^{\circ} \mathrm{C}\right.$ higher $)$. This unusual phenomenon could be contributed to fewer $\mathrm{H}_{2} \mathrm{O}$ molecules remaining in the film. Generally, $\mathrm{H}_{2} \mathrm{O}$ molecules could form hydrogen bonding with two groups in CS, i.e.., amino and hydroxyl groups, while the latter one has a stronger interaction with $\mathrm{H}_{2} \mathrm{O} .{ }^{[33]}$ It is reported that when the number of $\mathrm{H}_{2} \mathrm{O}$ molecules decreases in the sample, the hydrogen bonding is mainly found between amino groups and $\mathrm{H}_{2} \mathrm{O}$. However, the number of amino groups decreased after cross-linking, and $\mathrm{H}_{2} \mathrm{O}$ then turned to form hydrogen bonding with hydroxyl groups. Thus, the stronger interaction makes $\mathrm{H}_{2} \mathrm{O}$ harder to escape from the system. Another difference between the two is that for CS decomposition exothermic peak, the temperature of $1.5 \mathrm{~h}$ crosslinked film is lower than that of $0.5 \mathrm{~h}$ crosslinked film, which is consistent with Fig. 7. TGA and DTG curves of CS/PEO film and $1.5 \mathrm{~h}$ crosslinked CS/PEO film are also shown in Fig. 7. It could easily be drawn that after crosslinking modification, the evaporation peak of $\mathrm{H}_{2} \mathrm{O}$ (at 117.3 ${ }^{\circ} \mathrm{C}$ ) moved to a lower temperature zone (at 93.1 ${ }^{\circ} \mathrm{C}$ ), and so was the decomposition exothermic peak of CS (decreases from 282.3 to $269.1{ }^{\circ} \mathrm{C}$ ). These phenomena, together with the earlier DSC analysis in this study, proved the decrease of the thermal property of the blend film after cross-linking modification.

The surface morphologies of films are examined, and the results are listed in Fig. 8. A relatively even surface morphology is observed from neat CS film. But as PEO is introduced, an irregular pore structure is evolved and even maintained after the cross-linking modification, while the size of the pore structure becomes smaller. It is known that CS is a typical rigid molecule, and acicular crystallization can be formed under the effect of proper solvent. With the addition of $\mathrm{PEO}$, which is not perfectly compatible with CS, the pore structure is then formed when the PEO and CS phases migrated as the solvent evaporates. As glutaraldehyde is applied to achieve the cross-linking modification, the network structure arises as a result. This is the reason for the decreased pore size 

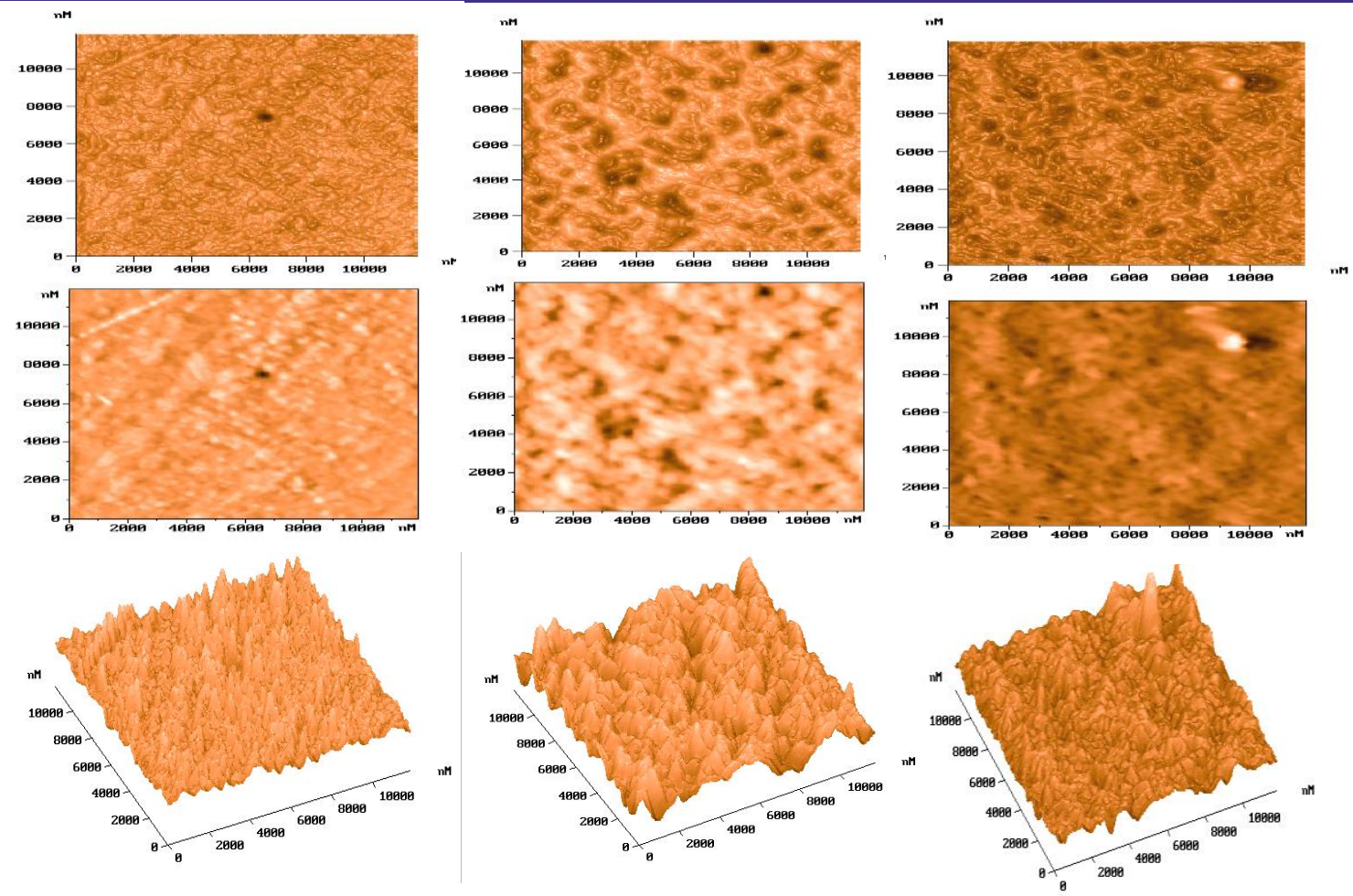

$\mathrm{RMS}=31.0 \mathrm{~nm}$

(a)

$\mathrm{RMS}=35.0 \mathrm{~nm}$

(b)
$\mathrm{RMS}=36.3 \mathrm{~nm}$

(c)

Fig. 8 AFM images of (a) CS membrane, (b) CS/PEO2000 membrane, (c) $1.5 \mathrm{~h}$ crosslinked CS/PEO2000 membrane. The DD of all CS is $86.11 \%$, the unit for $\mathrm{x}$ and $\mathrm{y}$ axis is $\mathrm{nm}$.

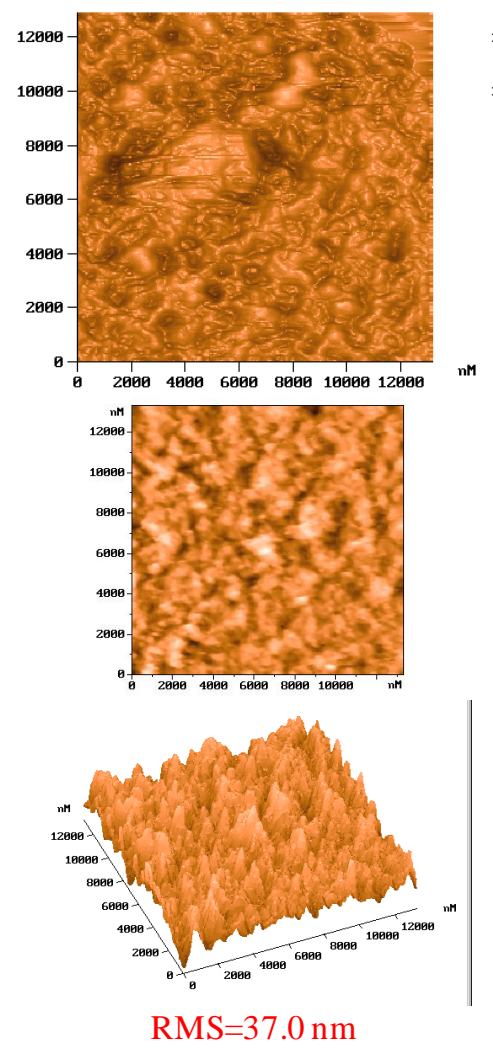

(a)
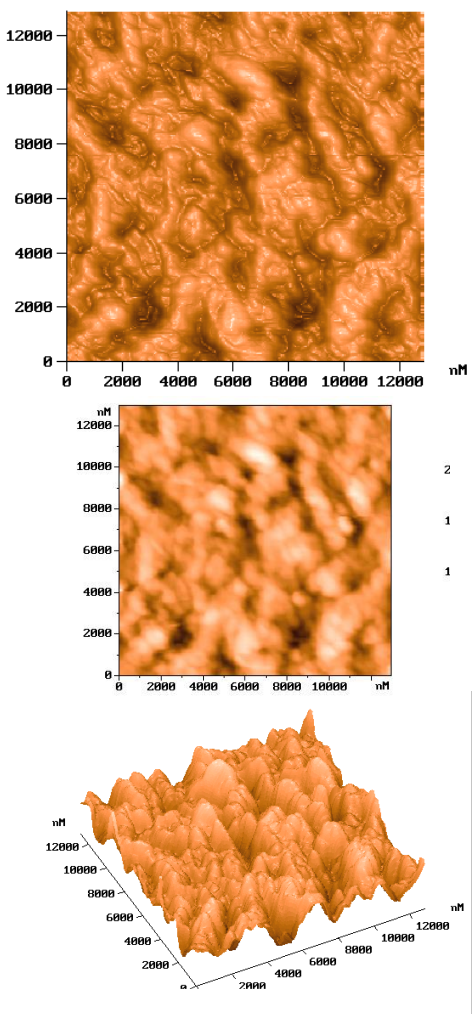

$\mathrm{RMS}=56.3 \mathrm{~nm}$

(b)

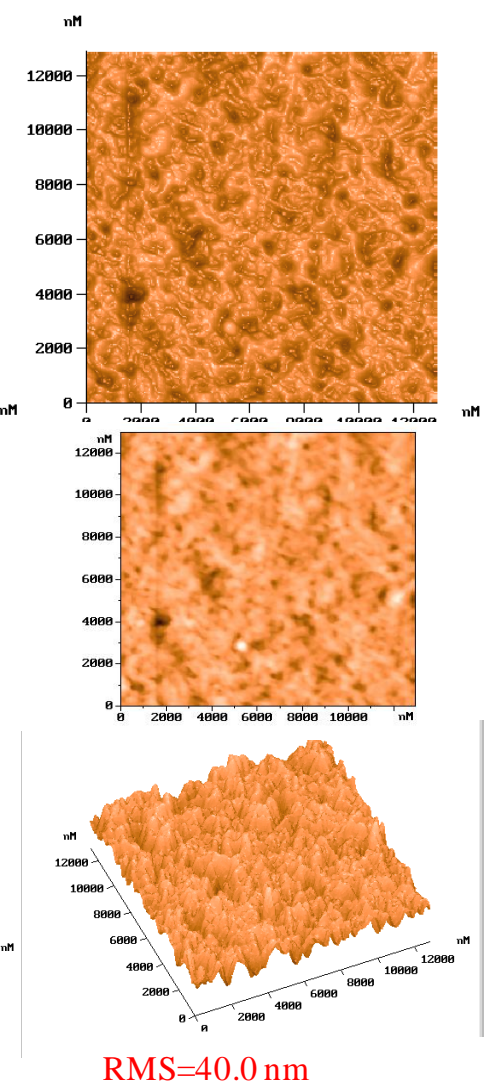

(c)

Fig. 9 AFM images of (a) $86.11 \% \mathrm{CS}$, (b) CS/PEO2000 membrane, (c) $1.5 \mathrm{~h}$ crosslinked CS/PEO2000 membrane after immersing in distilled water. The DD of all CS is $86.11 \%$, the unit for $\mathrm{x}$ and $\mathrm{y}$ axis is $\mathrm{nm}$. 
AFM is carried out by immersing the films in distilled water (Fig. 9). The sample must be processed by $0.5 \mathrm{~mL} \mathrm{NaCl}$ solution for $15 \mathrm{~min}$ to remove the remaining HAc solvent. According to the reports, ${ }^{[34]}$ a balance between PEO and $\mathrm{H}_{2} \mathrm{O}$ could be obtained after 2-min immersion, so we considered that PEO was completely removed after immersion. After immersion, the pores of CS/PEO film become much larger, resulting from the loss of PEO. While the surface structure of the crosslinked film just changed a little. Less PEO was lost because of the network structure, illustrating that the crosslinked film possesses a better ability to sustain its original surface structure when immersed in distilled water.

\subsection{Tensile strength of films}

Table 2. Tensile strength of different samples.

\begin{tabular}{c|c}
\hline Sample & Tensile strength (MPa) \\
\hline CS/PEO2000 & 37.59 \\
\hline $0.5 \mathrm{~h}$ crosslinked CS/PEO2000 & 38.02 \\
\hline 1h crosslinked CS/PEO2000 & 38.68 \\
\hline $1.5 \mathrm{~h}$ crosslinkedCS/PEO2000 & 36.97 \\
\hline CS/PEO400 & 33.55 \\
\hline $1.5 \mathrm{~h}$ crosslinked CS/PEO400 & 34.04 \\
\hline
\end{tabular}

The tensile strength data are shown in Table. 2 with the relative schematic diagram (Fig. S1). Prolonging the crosslinking time within a certain scope could achieve a better accomplished cross-linking reaction and higher cross-linking degree, which is quite crucial to the resulting properties of CS films. As shown in Table. 2, the tensile strength increases as the cross-linking degree increases. However, if the crosslinking reaction is proceeded further, the tensile strength decreases. When the cross-linking time is shorter, the crystallinity of the material increases, thus the tensile strength increases. However, the material is damaged to a certain extent and the tensile strength decreases as further increasing the cross-linking time. Besides, as seen in Table. 2 and Fig. S2, the tensile strength of the CS/PEO2000 film is generally larger than that of CS/PEO400. This may be because when the molecular weight of PEO is larger, there are more crosslinking points between macromolecules, resulting in an enhanced tensile strength.

\subsection{Water-absorbing property of films}

The influence of modification on the water-absorbing property is studied (Table 3). As the cross-linking time increases, the cross-linking degree is improved, while the water-absorbing amount gets decreased. This could easily be explained by the network structure formed after cross-linking. This structure reduces the tension of the system, impeding $\mathrm{H}_{2} \mathrm{O}$ molecules from penetration.

Table 3. Water absorption of CS / PEO2000 (DD = 86.11\%) samples at different cross-linking time.

\begin{tabular}{c|c|c|c|c}
\hline $\begin{array}{c}\text { cross-linking } \\
\text { time }(\mathrm{h})\end{array}$ & $W_{d}(\mathrm{~g})$ & $W_{s}(\mathrm{~g})$ & $\begin{array}{c}\text { Water absorbing } \\
\text { amount }(\%)\end{array}$ & $\begin{array}{c}\text { Cross-linking } \\
\text { density }\left(\times 10^{-4}\right)\end{array}$ \\
\hline 0.5 & 0.0413 & 0.1037 & 60.2 & 1.02 \\
\hline 1.5 & 0.0321 & 0.0775 & 58.6 & 1.57 \\
\hline 2 & 0.0381 & 0.0637 & 40.2 & 12.05 \\
\hline
\end{tabular}

\section{Conclusion}

PEO and glutaraldehyde were applied to modify CS films, and the properties of resultant films were examined. Benign physical blending was realized between CS and PEO through the intermolecular hydrogen bonding. The amino moieties of CS could crosslink with glutaraldehyde when CS/PEO film was processed with glutaraldehyde evaporation. AFM microphotographs demonstrated that the introduction of PEO had generated irregular pore structures, while the network structure took place after cross-linking modification and the pores became smaller. Tensile strength could be improved by cross-linking within a certain reaction time. Lastly, crosslinking modification led to a slight decrease in both waterabsorbing amount and thermal stability.

\section{Acknowledgements}

Financial support on this study is highly appreciated by the Opening Project of Key Laboratory of Biomedical Polymers of the Ministry of Education, Wuhan University (20070402), and Science and Technology Innovation Program of Harbin Institute of Technology.

\section{Supporting Information}

Applicable, https://dx.doi.org/10.30919/esmm5f780

\section{Conflict of interest}

There are no conflicts to declare.

\section{References}

[1] M. Miya, R. Iwamoto, S. Mima, J. Polym. Sci. Polym. Phys. Ed., 1984, 22, 1149, doi: 10.1002/pol.1984.180220615.

[2] T. Uragami, F. Yoshida, M. Sugihara, J. Appl. Polym. Sci. 1983, 28, 1361, doi: 10.1002/app.1983.070280410.

[3] T. Chandy, C.P. Sharma, J. Appl. Polym. Sci. 1992, 44, 2145, doi: https://doi.org/10.1002/app.1992.070441210.

[4] Y. Sawayanagi, N. Nimbu, T. Nagai, Chem. Pham. Bull., 1983, 31, 2507, doi: 10.1248/cpb.31.1980.

[5] H. Sashiwa, N. Yamamori, Y. Ichinose. Macromol. Biosci., 2003, 3, 231, doi: 10.1002/mabi.200390029. 
[6] S. Hirano, Y. Yamaguchi, M. Kamiya. Macromol. Biosci., 2003, 3, 629, doi: 10.1002/mabi.200350029.

[7] S. Lu, X. Song, D. Cao. J. Aool. Polym. Sci., 2004, 91, 3497, doi: 10.1002/app.13537.

[8] H. Sashiwa, N. Kawasaki, A. Nakayama. Biomacromolecules. 2002, 3, 1126, Doi: 10.1021/bm0200480.

[9] S. Fuentes, Biomavromolecules, 2000, 2, 239, doi: 10.1021/bm0055091.

[10] Y. Wan, H. Wu, A. X. Biomacromolecules, 2006, 4, 1362, doi: $10.1021 / \mathrm{bm} 0600825$.

[11] P. Kolhe, R. Kannan, Biomacromolecules, 2003, 1, 173, doi: $10.1021 / \mathrm{bm} 025689+$.

[12] T. Chandy, C.P. Sharma, Biomat. Art. Cells \& Art. Org., 1990, 18, 1, doi: 10.3109/10731199009117286.

[13] N. Kubota, K. Ohga, M. moriguchi, J. Appl. Polym. Sci., 1991, 42, 495, doi: 10.1002/app.1991.070420222.

[14]. D.K. Singh, A.R. Ray, J. Appl. Polym. Sci., 1994, 53, 1115, Doi: 10.1002/app.1994.070530814..

[15] D.K. Singh, A.R. Ray, J. Appl. Polym. Sci., 1997, 66, 869, doi: $\quad$ 10.1002/(SICI)1097-4628(19971031)66:5<869::AIDAPP7>3.0.CO;2-T.

[16] T. Sakaguchi, T. Horikoshi, A. Nakajima, A Gric. Biol. Chem. 1981, 7, 445, doi: 10.1080/00021369.1981.10864862.

[17] N. Nishi, A. Ebina, S. Nishimura, Int. J. Biol. Macromol. 1986, 9, 311, doi: 10.1016/0264-410X(85)90127-6.

[18] T. Tojimaa, H. Katsuraa, M. Nishikia, N. Nishi, S. Tokura, N. Sakairi, Carbohydr. Polym., 1999, 40, 17, doi: 10.1016/S01448617(99)00030-2.

[19] J. Jin, M. Song, J. Appl. poly., 2005, 6, 436, doi: 10.1002/app.24110.

[20] C. Knill, J. Kennedy, J. Mistry, Carbohyd.. Polym., 2004, 9, 55, doi: 10.1016/j.carbpol.2003.08.004.

[21] G. Fundueanu,M. Constantin,A. Dalpiaz, Carbohyd. Polym., 2004, 2, 159, doi: 10.1016/s0142-9612(03)00477-0.
[22] C. Luftensteiner, I.Schwendenwein, B. Paul, Carbohyd. Polym., 1999, 5, 35, doi: 10.1016/S0168-3659(98)00098-4.

[23] B. Tanima, M. Suamita, K. Singh, Int. J.Pharm., 2002, 122, 93, doi: 10.1016/S0378-5173(02)00267-3.

[24] R. Anandrao, I. Vijaykumar, H. W. Sung, Macromole. Biosci., 2005, 7, 112, doi: 10.1002/mabi.200500048.

[25] Y.L. Liu, C.Y. Hsu, J.Y. Lai. Biomacromolecules, 2005, 6, 368, doi: https://doi.org/10.1021/bm049531w.

[26] M.C. Gutierrez, M. Jobbagy, M.L. Ferrer. Chem. Mater., 2008, 20, 11, doi: 10.1021/bm049531w.

[27] L. Ding, C. Hao, Y. Xue. Biomacromolecules, 2007, 8, 1341, doi: $10.1021 / \mathrm{bm} 061224 \mathrm{y}$.

[28] Z. Hu, W.L. Chan, Y.S. Szeto. J. Appl. Polym. Sci., 2008,108, 52, doi: 10.1002/app.26822.

[29] C. Neto, J. Giacometti, A. ob, Carboh. Polym., 2005, 2, 62, Doi: 10.1016/j.carbpol.2005.02.022.

[30] B. Lee, J. Dalsin, P. Messersmith, Biomacromolecules, 2002, 3, 1038, doi: 10.1021/bm025546n.

[31] J. Yu, H. Du, Y.H. Zheng, J. of Wuhan Univ. (Nat. Sci. Ed.), 1999, 45, 440, doi: 10.1007/BF02832288.

[32] I. Glicokabir, A. Penhasi, K. Rubinstein, Carbohydr. Res., 1999, 316, 6, doi: 10.1016/S0008-6215(99)00025-7.

[33] A. E. Jobb, F.C. Ferreirab, J.L.C. Fonsecaa, Carbohydr. Polym., 2001, 7, 100, , doi: 10.1016/j.carbpol.2005.02.022.

[34] P. Sutandar, D. J. Ahn, E.I. Franses, Macromolecules, 1994, 27, 7316, doi: 10.1021/ma00103a013.

Publisher's Note Engineered Science Publisher remains neutral with regard to jurisdictional claims in published maps and institutional affiliations. 